Geom. Funct. Anal. Vol. 19 (2009) 1081-1100

DOI 10.1007/s00039-009-0026-2

Published online November 3, 2009

(C) 2009 The Author(s)

GAFA Geometric And Functional Analysis

This article is published with open access at Springerlink.com

\title{
POLYGONS IN BUILDINGS AND THEIR REFINED SIDE LENGTHS
}

\author{
Michael Kapovich, Bernhard Leeb and John J. Millson
}

\begin{abstract}
As in a symmetric space of noncompact type, one can associate to an oriented geodesic segment in a Euclidean building a vector valued length in the Euclidean Weyl chamber $\Delta_{e u c}$. In addition to the metric length it contains information on the direction of the segment. In this paper we study restrictions on the $\Delta_{\text {euc }}$-valued side lengths of polygons in Euclidean buildings. The main result is that for thick Euclidean buildings $X$ the set $\mathcal{P}_{n}(X)$ of possible $\Delta_{\text {euc }}$-valued side lengths of oriented $n$-gons depends only on the associated spherical Coxeter complex. We show moreover that it coincides with the space of $\Delta_{\text {euc }}$-valued weights of semistable weighted configurations on the Tits boundary $\partial_{T i t s} X$.

The side lengths of polygons in symmetric spaces of noncompact type are studied in the related paper [KLM1]. Applications of the geometric results in both papers to algebraic group theory are given in [KLM2].
\end{abstract}

\section{Introduction}

For a noncompact symmetric space of rank one, such as a hyperbolic plane, the only isometry invariant of a geodesic segment is its metric length. In a symmetric space of noncompact type and arbitrary rank the equivalence classes of oriented segments modulo the identity component of the isometry group are parameterized by the Euclidean Weyl chamber $\Delta_{e u c}$. We call the vector $\sigma(\gamma) \in \Delta_{\text {euc }}$ corresponding to an oriented segment $\gamma$ its $\Delta$-length. The same notion of $\Delta$-length can be defined in a Euclidean building, see section 3.1. Note that the directional part of the $\Delta$-length of a segment depends on its orientation. This is because the antipodal involution of the spherical Coxeter complex induces an, in general non-trivial, involutive selfisometry of the spherical Weyl chamber $\Delta_{s p h} \cdot\left(\Delta_{e u c}\right.$ is the complete Euclidean cone over $\left.\Delta_{s p h} \cdot\right)$

For a Euclidean building or a symmetric space of noncompact type $X$ we denote by $\mathcal{P}_{n}(X) \subset \Delta_{\text {euc }}^{n}$ the set of $\Delta$-side lengths which occur for oriented $n$-gons in $X$.

Keywords and phrases: Buildings, triangles, semistable configurations

2000 Mathematics Subject Classification: 53C21, 53C20, 51E24, 20E42 
Main Theorem 1.1. For a thick Euclidean building $X$ the set $\mathcal{P}_{n}(X)$ of possible $\Delta$-side lengths of oriented $n$-gons depends only on the spherical Weyl chamber $\Delta_{s p h}$ associated to $X$.

In other words, for any two thick Euclidean buildings an isomorphism of spherical Coxeter complexes, respectively, an isometry of spherical Weyl chambers induces an isometry of $\Delta$-side length spaces. In particular, automorphisms of Coxeter complexes induce self-isometries.

Our proof of the main theorem uses a relation between polygons in Euclidean buildings and weighted configurations on their spherical Tits buildings at infinity via a Gauss map type construction, see section 4.2. A weighted configuration on a spherical building $B$ is a map $\psi:(\mathbb{Z} / n \mathbb{Z}, \nu) \rightarrow B$ from a finite measure space. By composing $\psi$ with the natural projection $B \rightarrow \Delta_{s p h}$ onto the associated spherical Weyl chamber one obtains a map $(\mathbb{Z} / n \mathbb{Z}, \nu) \rightarrow \Delta_{s p h}$. We call the corresponding point in $\Delta_{\text {euc }}^{n}$ the $\Delta$-weights of the configuration $\psi$.

In order to characterize the weighted configurations on $\partial_{\text {Tits }} X$ which arise as Gauss maps of polygons in $X$, we introduce in section 4.1 a notion of (semi)stability for weighted configurations on abstract spherical buildings, see also [KLM1, §3.6]. It is motivated by Mumford stability in geometric invariant theory as explained in $[\mathrm{KLM} 1, \S 4]$. If $\psi$ is a weighted configuration on $\partial_{\text {Tits }} X$ then one can associate to it a natural convex function on $X$, the weighted Busemann function $b_{\psi}$ (well-defined up to an additive constant), and (semi)stability of $\psi$ amounts to certain asymptotic properties of $b_{\psi}$.

Theorem 1.2. Let $X$ be a Euclidean building. Then for $h \in \Delta_{\text {euc }}^{n}$ there exists an oriented $n$-gon in $X$ with $\Delta$-side lengths $h$ if and only if there exists a semistable weighted configuration on $\partial_{\text {Tits }} X$ with $\Delta$-weights $h$.

Balser $[\mathrm{Ba}]$ proves the sharper result that the weighted configurations on $\partial_{\text {Tits }} X$ which arise as Gauss maps of polygons in $X$ are precisely the semistable ones.

The proof of Theorem 1.2 relies upon the following result of independent interest, which is a generalization of Cartan's fixed-point theorem in the context of weak contractions of Hadamard spaces (see Lemma 4.6):

Theorem 1.3. Let $Y$ be a Hadamard space and $\Phi: Y \rightarrow Y$ a 1-Lipschitz self map. If the forward orbits $\left(\Phi^{n} y\right)_{n \geq 0}$ are bounded then $\Phi$ has a fixed point in $Y$.

Note that every spherical building is the Tits boundary of a Euclidean building, for instance, of the complete Euclidean cone over itself. As a step in our proof of the above results we obtain

Theorem 1.4. For a thick spherical building $B$ the set of possible $\Delta$-weights which occur for semistable weighted configurations only depends on the associated spherical Weyl chamber $\Delta_{s p h}$.

In our (logically independent) paper [KLM1] we investigate the $\Delta$-side lengths of polygons in symmetric spaces of noncompact type. We show there that Theorem 1.2 holds also in that case. Theorem 1.4 then implies that Theorem 1.1 holds for symmetric spaces of noncompact type, too. 
As a consequence of the results above and in [KLM1] it makes sense to denote by $\mathcal{P}_{n}\left(\Delta_{\text {sph }}\right) \subset \Delta_{\text {euc }}^{n}$ the space of $\Delta$-side lengths of oriented $n$-gons in thick Euclidean buildings or noncompact symmetric spaces with spherical Weyl chamber isometric to $\Delta_{s p h}$. It coincides with the space of $\Delta$-weights of semistable configurations on thick spherical buildings with this Weyl chamber.

In most cases the spaces $\mathcal{P}_{n}\left(\Delta_{\text {sph }}\right)$ are known to be finite-sided convex polyhedral cones, namely for spherical Coxeter complexes which occur for a symmetric space of noncompact type. As shown in [OS] and [KLM1] with rather different methods, $\mathcal{P}_{n}\left(\Delta_{\text {sph }}\right)$ can then be described as the solution set to a finite system of homogeneous linear inequalities. The system can be given explicitly in terms of the Schubert calculus on Grassmann manifolds associated to the symmetric spaces. The case of spherical Coxeter complexes, which occur for thick spherical buildings but not for symmetric spaces of noncompact type, is not covered and convexity is unknown.

So far we discussed $\Delta$-side lengths. In the case of Euclidean buildings there is a finer invariant for oriented geodesic segments taking values in $(E \times E) / W_{\text {aff }}$ where $\left(E, W_{a f f}\right)$ denotes the Euclidean Coxeter complex attached to $X$. We call it the refined length. Unlike the $\Delta$-length it also keeps track of the location of the endpoints of the segment. In cases when the affine Weyl group acts transitively, for example, for the affine Weyl group of symmetric spaces or ultralimits of thick Euclidean buildings with cocompact affine Weyl group, cf. [KlL], $\Delta$-length and refined length contain the same information.

An important step in our proof of the Main Theorem is a result concerning refined side lengths, namely the observation that polygons can be transferred between thick Euclidean buildings with isomorphic Euclidean Coxeter complexes while keeping their refined side lengths fixed; compare with Theorem 3.2.

Theorem 1.5. For a thick Euclidean building $X$ the set $\mathcal{P}_{n}^{r e f}(X) \subset\left((E \times E) / W_{\text {aff }}\right)^{n}$ of possible refined side lengths for $n$-gons in $X$ depends only on the associated Euclidean Coxeter complex $\left(E, W_{\text {aff }}\right)$.

More generally, polygons can be transferred to Euclidean buildings with larger affine Weyl groups while transforming their refined side lengths accordingly (Addendum 3.3), for instance, from a Euclidean building with one vertex to any other thick Euclidean building with isomorphic spherical Coxeter complex. Along the way we prove analogous results for polygons in spherical buildings.

The further study of the refined side length spaces $\mathcal{P}_{n}^{r e f}\left(\left(E, W_{\text {aff }}\right)\right)$ is relevant for certain applications to algebraic group theory and will be taken up in [KLM2]. More specifically, $\mathcal{P}_{n}^{r e f}\left(\left(E, W_{a f f}\right)\right)$ relates to the following algebraic problem.

Let $\mathcal{K}$ be a complete nonarchimedean valued field with the valuation $\operatorname{ring} \mathcal{O}$. Let $\underline{G}$ be a split reductive group over $\mathbb{Q}$.

Problem: Invariant factors of a product. Set $G:=\underline{G}(\mathcal{K})$ and $K:=\underline{G}(\mathcal{O})$. Give necessary and sufficient conditions on

$$
\alpha, \beta, \gamma \in K \backslash G / K
$$

in order that there exist elements $A, B, C \in G$ whose projections to $K \backslash G / K$ are 
$\alpha, \beta$ and $\gamma$, respectively, so that

$$
A B C=1 .
$$

There is a similar algebraic problem for the groups $G=\underline{G}(\mathcal{F})$, where $\mathcal{F}=\mathbb{R}$ or $\mathbb{C}$. The results of the present papers are used in [KLM2] to relate and to describe the solution sets of these algebraic problems. We refer the reader to [KLM2] for details.

Acknowledgements. During the work on this paper the first author was visiting the Max Plank Institute in Bonn and in Leipzig, he was also supported by the NSF grants DMS-02-03045 and DMS-04-05180. The first and the third authors were jointly supported by the NSF grant DMS-05-54349. The second author gratefully acknowledges financial support by the Deutsche Forschungsgemeinschaft (DFG-project LE 1312/2). The third author was supported by the Simons Foundation. The authors gratefully acknowledge support of these institutions. The authors are grateful to the referees for useful suggestions.

\section{Preliminaries}

In this section we will briefly review some basic facts about singular spaces with upper curvature bound, in particular with nonpositive curvature, and about Euclidean and spherical buildings. We will omit most of the proofs. For more details on singular spaces we refer to [B, Ch. 1-2], [BuBI, Ch.4+9], [KlL, Ch.2] and [L, Ch.2], and for the theory of buildings from a geometric viewpoint, i.e. within the framework of spaces with curvature bounded above, to [KlL, Ch. 3-4].

\subsection{Singular spaces with curvature bounded above.}

Upper curvature bounds. Let $k \in \mathbb{R}$. We denote by $M_{k}^{2}$ the model space of constant curvature $k$, i.e. the complete simply-connected Riemannian 2-manifold with constant sectional curvature $k$.

Let $Y$ be a complete metric space, not necessarily locally compact, with the property that any two points with distance $<\operatorname{diam}\left(M_{k}^{2}\right)$ are connected by a minimizing geodesic segment. (If $k \leq 0$ this means that $Y$ is a geodesic metric space.) A minimizing geodesic segment connecting $x$ and $y$ will be denoted by $\overline{x y}$. Note that this is an abuse of notation since there may be more than one minimizing geodesic segment connecting $x$ and $y$.

One can define curvature bounds for such metric spaces by comparison with the model spaces of constant curvature. For instance, one can compare the thickness of geodesic triangles. In this context, triangles are one-dimensional objects. A triangle $\Delta=\Delta(x, y, z)$ in $Y$ with vertices $x, y$ and $z$ is the union of three minimizing geodesic segments $\overline{x y}, \overline{y z}$ and $\overline{z x}$. A comparison triangle $\tilde{\Delta}=\Delta(\tilde{x}, \tilde{y}, \tilde{z})$ for $\Delta$ in $M_{k}^{2}$ is a triangle with the same side lengths. Every point $p$ on $\Delta$ corresponds to a point $\tilde{p}$ on $\tilde{\Delta}$ dividing the corresponding side in the same ratio, and one says that $\Delta$ is thinner than $\tilde{\Delta}$ if for any points $p, q$ on $\Delta$ the chord comparison inequality $d(p, q) \leq d(\tilde{p}, \tilde{q})$ holds. The space $Y$ has curvature $\leq k$ (globally) and is called a $\operatorname{CAT}(k)$-space if all 
geodesic triangles with diameter $<2 \operatorname{diam}\left(M_{k}^{2}\right)$ are thinner than their comparison triangles in $M_{k}^{2}$. It follows directly from the definition that every locally geodesic path of length $\leq \operatorname{diam}\left(M_{k}^{2}\right)$ in a $\operatorname{CAT}(k)$ space is a geodesic segment. Moreover, any two points with distance $<\operatorname{diam}\left(M_{k}^{2}\right)$ are connected by a unique minimizing geodesic segment.

A complete simply-connected Riemannian manifold with sectional curvatures $\leq k \leq 0$ has curvature $\leq k$ in the distance comparison sense.

Angles and spaces of directions. The presence of a curvature bound allows us to define angles between unit speed geodesic segments $\sigma_{1}, \sigma_{2}:[0, \epsilon) \rightarrow Y$ with the same initial point $\rho_{1}(0)=\rho_{2}(0)=y$, see e.g. [B, $\left.\S \mathrm{I} .3\right]$.

Suppose that $Y$ is a $\operatorname{CAT}(k)$ space. Let $\tilde{\alpha}(t)$ be the angle of a comparison triangle for $\Delta\left(y, \sigma_{1}(t), \sigma_{2}(t)\right)$ in $M_{k}^{2}$ at the vertex corresponding to $y$. Then the comparison angle $\tilde{\alpha}(t)$ is (weakly) monotonically decreasing as $t \searrow 0$. It therefore converges and we define the angle $\angle_{y}\left(\sigma_{1}, \sigma_{2}\right)$ as the limit. In this way one obtains a pseudo-metric on the space of unit speed geodesic segments emanating from a point $y \in Y$. The metric space $\left(\Sigma_{y} Y, \angle_{y}\right)$ obtained by identifying segments with angle zero and passing to the metric completion is called the space of directions at $p$. In the smooth case, $\Sigma_{y} Y$ is the unit tangent sphere. It turns out that in general $\Sigma_{y} Y$ is a CAT(1)-space, see e.g. [BrH, Ch. II, Th. 3.19] or [KlL, §2.1.3].

If $\Delta(x, y, z)$ is a geodesic triangle and $\tilde{\Delta}(\tilde{x}, \tilde{y}, \tilde{z})$ is a comparison triangle, the angle comparison $\angle_{x}(y, z) \leq \angle_{\tilde{x}}(\tilde{y}, \tilde{z})$ holds as a consequence of the definition of angles, see [B, Ch. I, Prop. 3.13].

2.2 Hadamard spaces. We will be mainly interested in CAT(0)-spaces. These are also called Hadamard spaces since they generalize Hadamard manifolds which are defined to be complete simply-connected Riemannian manifolds of nonpositive curvature. Symmetric spaces of noncompact type are Hadamard manifolds and Euclidean buildings are singular (i.e. non-Riemannian) Hadamard spaces, see e.g. $[\mathrm{E}],[\mathrm{KlL}],[\mathrm{D}]$. All Hadamard spaces considered in this paper are assumed to be metrically complete.

A basic consequence of the CAT(0)-property is the convexity of the distance function, i.e. for any two constant speed geodesic segments $\sigma_{1}, \sigma_{2}:[a, b] \rightarrow X$ in a Hadamard space $X$ the distance $t \mapsto d\left(\sigma_{1}(t), \sigma_{2}(t)\right)$ between fellow travellers is a convex function, see e.g. [B, Ch. I, Prop. 5.4]. It follows that any two points can be connected by a unique geodesic segment. In particular, Hadamard spaces are contractible.

Boundary at infinity. A geodesic ray is an isometric embedding $\rho:[0, \infty) \rightarrow X$. By abusing notation, we will frequently identify geodesic rays with their images. We say that two rays are asymptotic if they have bounded Hausdorff distance from each other or, equivalently, if the convex function $t \mapsto d\left(\rho_{1}(t), \rho_{2}(t)\right)$ is bounded and hence nonincreasing. Asymptoticity is an equivalence relation, and the set of equivalence classes of geodesic rays is called the ideal boundary or boundary at infinity $\partial_{\infty} X$ of $X$. An element $\xi \in \partial_{\infty} X$ is an ideal point or a point at infinity. A ray representing 
$\xi$ is said to be asymptotic to $\xi$. We will use the notation $\overline{x \xi}$ to denote the unique geodesic ray from $x \in X$ asymptotic to $\xi \in \partial_{\infty} X$; see e.g. [B, Ch. II, Lem. 2.1].

The ideal boundary $\partial_{\infty} X$ carries a natural topology, called cone topology, which will however not play a big role in this paper. A basis for the cone topology is given by subsets of the following form: For a ray $\rho_{0}:[0, \infty) \rightarrow X$ and numbers $l, \epsilon>0$ consider all ideal points in $\partial_{\infty} X$ which are represented by rays $\rho:[0, \infty) \rightarrow X$ such that $d\left(\rho(t), \rho_{0}(t)\right)<\epsilon$ for $0 \leq t<l$.

More important for us will be a natural metric on $\partial_{\infty} X$, the Tits metric. Given two ideal points $\xi_{1}, \xi_{2} \in \partial_{\infty} X$ we pick geodesic rays $\rho_{1}, \rho_{2}:[0, \infty) \rightarrow X$ representing them and a point $x \in X$. In analogy with the definition of angles above, let $\tilde{\alpha}(t)$ be the angle of a comparison triangle for $\Delta\left(\rho_{1}(t), x, \rho_{2}(t)\right)$ at the vertex corresponding to $x$. The $\operatorname{limit}_{t \rightarrow \infty} \tilde{\alpha}(t)$ always exists. It depends only on $\xi_{1}$ and $\xi_{2}$, and not on the representing rays $\rho_{1}, \rho_{2}$ and the base point $x$, see e.g. [B, Ch. II, Th. 4.4]. The Tits (angular) distance $\angle_{\text {Tits }}\left(\xi_{1}, \xi_{2}\right)$ is defined as this limit, i.e.

$$
2 \sin \frac{\angle_{\text {Tits }}\left(\xi_{1}, \xi_{2}\right)}{2}=\lim _{t \rightarrow \infty} \frac{d\left(\rho_{1}(t), \rho_{2}(t)\right)}{t} .
$$

The definition implies the useful inequality

$$
\angle_{x}(\xi, \eta) \leq \angle_{\text {Tits }}(\xi, \eta)
$$

for all $x \in X$. The metric space $\partial_{\text {Tits }} X=\left(\partial_{\infty} X, \angle_{\text {Tits }}\right)$ is called the Tits boundary. Note that the Tits distance $\angle_{\text {Tits }}$, as defined here, does in general not coincide with the length metric associated to it - some authors call the latter one Tits metric - but it does so if $X$ is a symmetric space or a Euclidean building. As for the spaces of directions, it turns out that the Tits boundary is a CAT(1)-space, see e.g. [B, Ch. II, Cor. 4.9]. Note that the Tits metric does in general not induce the cone topology. The Tits metric is lower semicontinuous with respect to the cone topology and induces a topology which is (in general strictly) finer than the cone topology.

Busemann functions. Busemann functions measure the relative distance from points at infinity. They are constructed as follows. For an ideal point $\xi \in \partial_{\infty} X$ and a ray $\rho:[0, \infty) \rightarrow X$ asymptotic to it we define the Busemann function $b_{\xi}$ as the pointwise monotone limit

$$
b_{\xi}(x):=\lim _{t \rightarrow \infty}(d(x, \rho(t))-t)
$$

of normalized distance functions. One checks that, up to an additive constant, $b_{\xi}$ does not depend on the chosen ray $\rho$. As a limit of distance functions $b_{\xi}$ is convex and 1-Lipschitz continuous, see e.g. [BrH, Ch. II, Prop. 8.22]. The level and sublevel sets of Busemann functions are called horospheres, respectively, horoballs.

Convex functions have directional derivatives. For Busemann functions they are given by the formula

$$
\frac{d}{d t^{+}}\left(b_{\xi} \circ \sigma\right)(t)=-\cos \angle_{\sigma(t)}\left(\sigma^{\prime}(t), \xi\right)
$$


where $\sigma: I \rightarrow X$ is a unit speed geodesic segment and the angle on the right-hand side is taken between the positive direction $\sigma^{\prime}(t) \in \Sigma_{\sigma(t)} X$ of the segment $\sigma$ at $\sigma(t)$ and the ray emanating from $\sigma(t)$ asymptotic to $\xi$. (The equality (2) easily follows from the first variation formula for the distance functions in Hadamard spaces, $[\mathrm{BrH}$, p. 185].)

Note that, by the definition, along a ray $\rho$ asymptotic to $\xi$ the Busemann function $b_{\xi}$ is affine linear, i.e. $b_{\xi}(\rho(t))=-t+$ const. As convex Lipschitz functions, Busemann functions are asymptotically linear along any ray $\rho$ and we define the asymptotic slope of $b_{\xi}$ at an ideal point $\eta \in \partial_{\infty} X$ by

$$
\operatorname{slope}_{\xi}(\eta)=\lim _{t \rightarrow \infty} \frac{b_{\xi}(\rho(t))}{t}
$$

for a ray $\rho$ asymptotic to $\eta$. Since $\angle_{\rho(t)}(\xi, \eta) \nearrow \angle_{\text {Tits }}(\xi, \eta)$ as $t \rightarrow \infty$, the formula (2) implies that

$$
\operatorname{slope}_{\xi}(\eta)=-\cos \angle_{\text {Tits }}(\xi, \eta) \text {. }
$$

Cones. Given a metric space with diameter $\leq \pi$ one constructs the complete Euclidean cone Cone $(B)$ over $B$ by mimicking the construction which produces Euclidean 3-space from the 2-dimensional unit sphere. The underlying set is $B \times[0, \infty) / \sim$ where $\sim$ collapses $B \times\{0\}$ to a point called the tip. For $v_{1}, v_{2} \in B$ and $t_{1}, t_{2} \geq 0$ we consider rays $\rho_{i}:[0, \infty) \rightarrow \mathbb{R}^{2}$ in Euclidean plane with the same initial point $o$ and angle $\angle_{o}\left(\rho_{1}, \rho_{2}\right)=d_{B}\left(v_{1}, v_{2}\right)$. We then define the distance of points in $\operatorname{Cone}(B)$ represented by $\left(v_{1}, t_{1}\right)$ and $\left(v_{2}, t_{2}\right)$ as $d_{\mathbb{R}^{2}}\left(\left(\rho_{1}\left(t_{1}\right), \rho_{2}\left(t_{2}\right)\right)\right.$.

The space $\operatorname{Cone}(B)$ is $\operatorname{CAT}(0)$ if and only if $B$ is $\operatorname{CAT}(1)$. In this case there is a natural isometry $B \cong \partial_{\text {Tits }} \operatorname{Cone}(B)$. See [BrH, Ch. II, Th. 3.14].

REMARK 2.1. The 0-dimensional sphere $S^{0}$ is declared to have diameter $\pi$, so that $\mathbb{R}$ is the Euclidean cone over $\mathrm{S}^{0}$.

2.3 Coxeter complexes. Our treatment of Coxeter complexes and buildings in this and the next section follows [KlL, Ch. 3-4].

Spherical Coxeter complexes. A spherical Coxeter complex is a pair $\left(S, W_{s p h}\right)$ where $S$ is a unit sphere and $W_{s p h}$ is a finite subgroup of $\operatorname{Isom}(S)$ generated by reflections. Here, by a reflection one means an involutive isometry with fixed-point set a great sphere of codimension one. $W_{s p h}$ is called the Weyl group and the fixed-point sets of the reflections in $W_{s p h}$ are called walls. The pattern of walls gives $S$ a natural structure of a (polysimplicial) cell complex $\Sigma$. This cell complex is also frequently referred to as a Coxeter complex. The top-dimensional cells of $\Sigma$, the chambers, are fundamental domains for the action $W_{s p h} \curvearrowright S$. They are spherical simplices if $W_{s p h}$ does not have a global fixed point in $S$. If convenient, we identify the spherical model Weyl chamber $\Delta_{s p h}=S / W_{\text {sph }}$ with one of the chambers in $S$.

An embedding $\left(S, W_{s p h}\right) \hookrightarrow\left(S^{\prime}, W_{s p h}^{\prime}\right)$ of spherical Coxeter complexes of equal dimensions consists of a reflection-preserving monomorphism $\iota: W_{s p h} \hookrightarrow W_{s p h}^{\prime}$ and an $\iota$-equivariant isometry $\alpha: S \rightarrow S^{\prime}$. The isometry $\alpha$ therefore maps walls to walls. We call the embedding of Coxeter complexes an isomorphism if $\iota$ is an isomorphism. 
Euclidean Coxeter complexes. A Euclidean Coxeter complex is a pair $\left(E, W_{a f f}\right)$, where $E$ is a Euclidean space and $W_{\text {aff }} \subset I \operatorname{som}(E)$ is a subgroup generated by reflections. Again reflection means a reflection at an affine hyperplane. We require moreover that the induced reflection group on the sphere at infinity $\partial_{\text {Tits }} E$ is finite.

One obtains the associated spherical Coxeter complex $\left(S, W_{s p h}\right)$. Here the unit sphere $S=\partial_{\text {Tits }} E$ is the ideal boundary of $E$ and $W_{s p h}:=\operatorname{rot}\left(W_{\text {aff }}\right)$, where

$$
\text { rot }: \operatorname{Isom}(E) \rightarrow I \operatorname{som}\left(\partial_{\text {Tits }} E\right)
$$

is the natural homomorphism mapping an affine transformation to its linear part. Let $\Delta_{s p h}$ be the spherical model Weyl chamber of $\left(S, W_{s p h}\right)$. We define the Euclidean model Weyl chamber $\Delta_{\text {euc }}$ of $\left(E, W_{\text {aff }}\right)$ as the complete Euclidean cone over $\Delta_{s p h}$, that is, $\Delta_{e u c}=\operatorname{Cone}\left(\Delta_{s p h}\right)$. It is canonically identified with the quotient of the vector space of translations on $E$ by the natural action of $W_{s p h}$ by conjugation and one has a well-defined addition and scalar multiplication by positive real numbers on $\Delta_{\text {euc }}$.

A wall in the Coxeter complex $\left(E, W_{a f f}\right)$ is an affine hyperplane fixed by a reflection in $W_{\text {aff. }}$ Singular subspaces of $\left(E, W_{\text {aff }}\right)$ are defined as intersections of walls, and vertices are zero-dimensional singular subspaces.

We denote the kernel of rot $: W_{\text {aff }} \rightarrow W_{\text {sph }}$ by $L=L_{\text {trans }}$ and we refer to it as the translation subgroup. The exact sequence $0 \rightarrow L \rightarrow W_{\text {aff }} \rightarrow W_{s p h} \rightarrow 1$ splits, i.e. the affine Weyl group decomposes as the semidirect product $W_{\text {aff }} \cong W_{s p h} \ltimes L$. The two extreme cases are

1. $L$ is the full group of translations on $E$, as it happens for the Euclidean Coxeter complex attached to a symmetric space of noncompact type.

2. $L=\{0\}$ and $W_{a f f}=W_{s p h}$ is finite as in the case of Euclidean buildings with one vertex.

A Euclidean Coxeter complex $\left(E, W_{\text {aff }}\right)$ is called discrete if $W_{\text {aff }}$ is a discrete subgroup of $\operatorname{Isom}(E)$. If moreover $W_{\text {aff }}$ acts cocompactly on $E$, the pattern of walls induces a natural structure of polysimplicial cell complex on $E$. The top-dimensional cells, the alcoves, are fundamental domains for the action $W_{\text {aff }} \curvearrowright E$ and the reflections at the faces of one cell generate the group $W_{\text {aff. }}$. The alcoves are canonically isometric to the model Weyl alcove $E / W_{\text {aff. }}$ The Weyl alcove is different from the Euclidean Weyl chamber.

An embedding $\left(E, W_{\text {aff }}\right) \hookrightarrow\left(E^{\prime}, W_{\text {aff }}^{\prime}\right)$ of Euclidean Coxeter complexes of equal dimensions consists of a reflection-preserving monomorphism $\iota: W_{\text {aff }} \hookrightarrow W_{\text {aff }}^{\prime}$ and an $\iota$-equivariant homothety $\alpha: E \rightarrow E^{\prime}$. The homothety $\alpha$ maps walls to walls. We call the embedding of Coxeter complexes an isomorphism if $\iota$ is an isomorphism. A dilation of a Coxeter complex $\left(E, W_{\text {aff }}\right)$ is a self-embedding such that the homothety $\alpha: E \rightarrow E$ is a dilation. 
2.4 Buildings. We refer the reader to [Bro], $[\mathrm{R}]$, $[\mathrm{KlL}]$ for the detailed discussion of spherical and Euclidean buildings. Here we present only a brief overview.

Spherical buildings. A spherical building modelled on a spherical Coxeter complex $\left(S, W_{s p h}\right)$ is a CAT(1)-space $B$ together with a maximal atlas of charts, i.e. isometric embeddings $S \hookrightarrow B$. The image of a chart is an apartment in $B$. We require that any two points are contained in a common apartment and that the coordinate changes between charts are induced by isometries in $W_{s p h}$.

ExAmple. Suppose that $B$ is a 0-dimensional spherical building modelled on the Coxeter complex $\left(\mathrm{S}^{0}, \mathbb{Z}_{2}\right)$. Then the metric space underlying $B$ is a discrete metric space where any two distinct points have distance $\pi$. Indeed, the 0-dimensional sphere $\mathrm{S}^{0}$ has diameter $\pi$. Pick two distinct points $\xi, \eta \in B$. Then there exists a chart $\varphi: \mathrm{S}^{0} \rightarrow B$ whose image contains $\{\xi, \eta\}$. Since $\varphi$ is an isometric embedding, the distance between $\xi$ and $\eta$ equals $\pi$.

We will often denote the metric on a spherical building by $\angle_{\text {Tits }}$ because in this paper spherical buildings usually arise as Tits boundaries.

The cell structure and the notions of wall, chamber etc. carry over from the Coxeter complex to the building. The building $B$ is called thick if every codimensionone face is adjacent to at least three chambers. A non-thick building can always be equipped with a natural structure of a thick building by reducing the Weyl group. Namely, pick an apartment $S \subset B$ and consider the collection $\mathcal{L}$ of those walls in $S$ which are adjacent to at least three half-apartments in $B$. Consider the subgroup $W^{\prime} \subset W$ generated by reflections in the walls which belong to $\mathcal{L}$. One can verify that the walls of the Coxeter complex $\left(S, W^{\prime}\right)$ belong to $\mathcal{L}$. Let $Y$ be the metric space underlying $B$. Now, define the new building $B^{\prime}$, whose underlying metric space is $Y$ and whose atlas consist of the old charts $\varphi:\left(S, W^{\prime}\right) \rightarrow B$. One can show that $B^{\prime}$ is a thick building modelled on $\left(S, W^{\prime}\right)$. See [S] and [KlL, §3.7] for details.

If $W_{s p h} \curvearrowright S$ does not have a global fixed point, the chambers of $B$ are spherical simplices and the building carries a natural structure as a piecewise spherical simplicial complex. We will then refer to the cells as simplices.

There is a canonical 1-Lipschitz continuous accordion map acc $: B \rightarrow \Delta_{\text {sph }}$ folding the building onto the model Weyl chamber so that every chamber projects isometrically. Namely, given a point $\xi \in B$, pick a chart $\varphi: S \rightarrow B$ whose image contains $\xi$. Then $\operatorname{acc}(\xi)$ is the projection of $\varphi^{-1}(\xi)$ to the model chamber $\Delta_{s p h}$ which we identify with $S / W_{s p h}$. The point $\operatorname{acc}(\xi)$ is called the type of the point $\xi \in B$, and a point in $B$ is called regular if its type is an interior point of $\Delta_{s p h}$.

Euclidean buildings. A Euclidean building modelled on a Euclidean Coxeter complex $\left(E, W_{\text {aff }}\right)$ is a $\mathrm{CAT}(0)$-space $X$ together with a maximal atlas of charts $E \hookrightarrow X$ subject to the following conditions: The charts are isometric embeddings, their images are called apartments; any pair of points and, more generally, any ray and any complete geodesic, is contained in an apartment; the coordinate changes between charts are restrictions of isometries in $W_{\text {aff. }}$ In addition, one imposes a certain angle rigidity condition on the spaces of directions $\Sigma_{x} X, x \in X$. See [KlL, p. 150] for details. 
A Euclidean building is called thick if every wall is an intersection of apartments. It is called discrete if it is modelled on a discrete Euclidean Coxeter complex. (The angle rigidity condition is automatic in this case.) It then carries a natural structure of a polyhedral cell complex.

If $X$ is a thick Euclidean building modelled on the Coxeter complex $\left(E, W_{a f f}\right)$ then its Tits boundary $\partial_{\text {Tits }} X$ is a thick spherical building modelled on $\left(\partial_{\text {Tits }} E, W_{s p h}\right)$. The spaces of directions $\Sigma_{x} X$ are spherical buildings modelled on $\left(\partial_{\text {Tits }} E, W_{s p h}\right)$, see [KlL, §4.4]. However, the building $\Sigma_{x} X$ is thick (with respect to this model spherical Coxeter complex) if and only if $x$ is special, i.e. corresponds in a chart to a point in $E$ with maximal possible stabilizer $\cong W_{s p h}$. Note that all directions $\xi \in \Sigma_{x} X$ are represented by geodesic segments initiating in $x$, i.e. in the construction of the space of directions in the case of Euclidean buildings (as for Hadamard manifolds) one does not have to pass to the metric completion, cf. section 2.1.

If $B$ is a spherical building then $X=\operatorname{Cone}(B)$ carries a natural induced Euclidean building structure. Namely: Since $B$ is a $\operatorname{CAT}(1)$ space, the cone $C o n e(B)$ is $\operatorname{CAT}(0)$. Apartments in $\operatorname{Cone}(B)$ are the cones of apartments in $B$. If $\left(S, W_{s p h}\right)$ is the Coxeter complex of $B$, then $\left(E, W_{s p h}\right)$ is the Coxeter complex for $X$, where we identify $W_{s p h}$ with the subgroup of $I \operatorname{som}(E)$ stabilizing the origin in $E$ (the point corresponding to the tip of the cone $X$ ). The building axioms for $X$ follow immediately from the building axioms for $B$. (Since the Coxeter complex $\left(E, W_{s p h}\right)$ is discrete, the angle rigidity axiom is immediate.) Note that the tip to the cone Cone $(B)$ is the unique vertex of $X$ and $B$ is isomorphic to $\partial_{\text {Tits }} X$ as a spherical building.

\section{Transfer of Polygons Between Buildings}

3.1 Polygons and side lengths. By an $n$-gon $z_{1} \ldots z_{n}$ in a metric space $Z$ we mean a map $\mathbb{Z} / n \mathbb{Z} \rightarrow Z$ carrying $i$ to the vertex $z_{i}$.

If $Z$ is a CAT(0)-space, such as a Euclidean building or a symmetric space of noncompact type, then any two points in $Z$ are connected by a unique geodesic segment and the polygon can be promoted to a 1-dimensional object. For any pair of successive vertices $x_{i-1}$ and $x_{i}$ one has a well-defined side $\overline{x_{i-1} x_{i}}$. If $Z$ is a CAT(1)-space, for instance a spherical building, one has well-defined sides for successive vertices of distance $<\pi$. The cyclic ordering of the vertices determines a natural orientation of the sides.

Let $\left(E, W_{a f f}\right)$ be a Euclidean Coxeter complex. To a pair of points $(p, q)$ in $E$ one can associate a vector in the Euclidean Weyl chamber $\Delta_{e u c}=\operatorname{Cone}\left(\Delta_{s p h}\right)$ as follows. The translations on the affine space $E$ form a vector space on which the spherical Weyl group $W_{s p h}$ acts by conjugation. The quotient can be canonically identified with $\Delta_{\text {euc }}$. Thus we can attach to $(p, q)$ the image in $\Delta_{\text {euc }}$ of the translation carrying $p$ to $q$. We call this vector $\sigma(p, q)$ the $\Delta$-length of the oriented geodesic segment $\overline{p q}$. It is invariant under isometries in $W_{\text {aff }}$ by construction. Note that the directional part of the $\Delta$-length depends on the orientation of the segment. The reason is that 
the antipodal involution of the spherical Coxeter complex $\left(\partial_{\text {Tits }} E, W_{s p h}\right)$ induces an in general non-trivial involutive self-isometry of the spherical Weyl chamber $\Delta_{s p h}$.

The complete invariant of a pair $(p, q)$ modulo the action of $W_{\text {aff }}$ is its image $\sigma_{r e f}(p, q)$ under the natural projection to $(E \times E) / W_{\text {aff. }}$. We call it the refined length of the oriented segment $\overline{p q}$. The $\Delta$-length is obtained by composing $\sigma_{\text {ref }}$ with the natural forgetful map $(E \times E) / W_{\text {aff }} \rightarrow \Delta_{\text {euc }}$. The $\Delta$-length contains the complete information about the metric length and the direction of the segment modulo the spherical Weyl group, while the refined length keeps track in addition of the location of the endpoints. If the affine Weyl group contains the full translation group, as in the case of the Euclidean Coxeter complex attached to a symmetric space of noncompact type, then $(E \times E) / W_{a f f} \cong \Delta_{\text {euc }}$ and $\Delta$-length and refined length contain the same information.

As in the Euclidean case, one can attach to a pair of points $(p, q)$ in a spherical Coxeter complex $(S, W)$ the refined length $\sigma_{r e f}(p, q) \in(S \times S) / W$, and it is invariant under the $W$-action.

These notions of length carry over to geometries modelled on Coxeter complexes. One chooses an apartment containing a given pair of points and measures length inside the apartment. The length is well-defined because the coordinate changes between apartment charts are restrictions of isometries in the Weyl group.

Hence one has the notion of $\Delta$-length in Euclidean buildings and symmetric spaces of noncompact type, and one has the notion of refined length in Euclidean and spherical buildings. Note that in a symmetric space of noncompact type, although well-defined, the notion of refined length does not give more information than the $\Delta$-length because the affine Weyl group acts transitively.

Let $X$ be a Euclidean building or a symmetric space of noncompact type and let $\left(E, W_{\text {aff }}\right)$ be its associated Euclidean Coxeter complex. To a polygon $x_{1} \ldots x_{n}$ in $X$ we associate its $\Delta$-side lengths $\left(\sigma\left(x_{0}, x_{1}\right), \ldots, \sigma\left(x_{n-1}, x_{n}\right)\right) \in \Delta_{\text {euc }}^{n}$ and its refined side lengths $\left(\sigma_{\text {ref }}\left(x_{0}, x_{1}\right), \ldots, \sigma_{r e f}\left(x_{n-1}, x_{n}\right)\right) \in\left((E \times E) / W_{\text {aff }}\right)^{n}$. Analogously one can attach refined side lengths with values in $((S \times S) / W)^{n}$ to $n$-gons in spherical buildings modelled on the Coxeter complex $(S, W)$.

Definition 3.1. We define $\mathcal{P}_{n}(X) \subset \Delta_{\text {euc }}^{n}$, respectively $\mathcal{P}_{n}^{r e f}(X) \subset\left((E \times E) / W_{\text {aff }}\right)^{n}$ as the space of possible $\Delta$-side lengths, respectively refined side lengths, which occur for $n$-gons in $X$.

3.2 The transfer argument. This section is devoted to the proof of Theorem 1.5 stated in the introduction. In fact we need to prove the same result for spherical buildings since we will proceed by induction on the dimension and apply the induction assumption to the spaces of directions. Recall that the spaces of directions of Euclidean or spherical buildings are spherical buildings, cf. section 2.4.

Transfer Theorem 3.2. (i) If $X$ and $X^{\prime}$ are thick Euclidean buildings modelled on the same Euclidean Coxeter complex $\left(E, W_{\text {aff }}\right)$ then $\mathcal{P}_{n}^{r e f}(X)=\mathcal{P}_{n}^{r e f}\left(X^{\prime}\right)$.

(ii) The analogous assertion holds for thick spherical buildings modelled on the same spherical Coxeter complex. 
In other words, isomorphisms of associated Coxeter complexes (cf. section 2.3) induce bijections of refined side length spaces.

Proof. (ii) We first discuss the spherical case. Let $B$ and $B^{\prime}$ be thick spherical buildings modelled on the same spherical Coxeter complex $(S, W)$. Given a polygon $P$ in $B$, we will transfer it to a polygon $P^{\prime}$ in $B^{\prime}$ while preserving the refined side lengths. It suffices to show the following assertion: (*) Let $\xi, \eta, \zeta \in B$ and $\xi^{\prime}, \zeta^{\prime} \in B^{\prime}$ so that the oriented segments $\overline{\xi \zeta}$ and $\overline{\xi^{\prime} \zeta^{\prime}}$ have equal refined lengths. Then there exists $\eta^{\prime} \in B^{\prime}$ so that the triangles $\Delta(\xi, \eta, \zeta)$ and $\Delta\left(\xi^{\prime}, \eta^{\prime}, \zeta^{\prime}\right)$ have the same refined side lengths.

We proceed by induction on the dimension of the buildings. The assertion $(*)$ is trivial in dimension 0 . We therefore assume that $\operatorname{dim}(B)=\operatorname{dim}(B)=d>0$ and that $(*)$ has been proven in dimensions $<d$.

There is a finite subdivision of the side $\overline{\xi \eta}$ by points $\xi_{0}=\xi, \xi_{1}, \ldots, \xi_{k-1}, \xi_{k}=\eta$ such that each geodesic triangle $\Delta\left(\zeta, \xi_{i}, \xi_{i+1}\right)$ is contained in an apartment. Namely, choose the subdivision so that each subsegment $\overline{\xi_{i} \xi_{i+1}}$ is contained in a chamber $\Delta_{i}$ and note that each chamber $\Delta_{i}$ is contained in an apartment through $\zeta$. (The analogous assertion for triangles in Euclidean buildings was proven in [KlL, Cor. 4.6.8].)

We need to find points $\xi_{1}^{\prime}, \ldots, \xi_{k}^{\prime}$ in $B^{\prime}$ such that the triangles $\Delta\left(\zeta^{\prime}, \xi_{i}^{\prime}, \xi_{i+1}^{\prime}\right)$ have the same refined side lengths as $\Delta\left(\zeta, \xi_{i}, \xi_{i+1}\right)$ for all $i$ and such that $\angle_{\xi_{i}^{\prime}}\left(\xi_{i-1}^{\prime}, \xi_{i+1}^{\prime}\right)=\pi$. This will be done by a second induction on $i$. We can choose $\xi_{1}^{\prime}$ in an apartment containing $\overline{\zeta^{\prime} \xi^{\prime}}$. Suppose that $\xi_{i}^{\prime}$ has been found, $i \geq 1$. In order to find the direction $\overrightarrow{\xi_{i}^{\prime} \xi_{i+1}^{\prime}}$ at $\xi_{i}^{\prime}$, we apply the induction hypothesis (of the first induction on the dimension) to the links $\Sigma_{\xi_{i}} B$ and $\Sigma_{\xi_{i}^{\prime}} B^{\prime}$, which are thick spherical buildings of dimension $d-1$ modelled on the same spherical Coxeter complex, and transfer the triangle $\Delta\left(\overrightarrow{\xi_{i} \xi_{i-1}}, \overrightarrow{\xi_{i} \zeta}, \overrightarrow{\xi_{i} \xi_{i+1}}\right)$ in $\Sigma_{\xi_{i}} B$ to a triangle $\Delta\left(\overrightarrow{\xi_{i}^{\prime} \xi_{i-1}^{\prime}}, \overrightarrow{\xi_{i}^{\prime} \zeta^{\prime}}, \overrightarrow{\xi_{i}^{\prime} \xi_{i+1}^{\prime}}\right)$ in $\Sigma_{\xi_{i}^{\prime}} B^{\prime}$ with the same refined side lengths. We then choose an apartment in $B^{\prime}$ which contains $\overline{\zeta^{\prime} \xi_{i}^{\prime}}$ and is tangent to the direction $\overline{\xi_{i}^{\prime} \xi_{i+1}^{\prime}}$. Inside this apartment there is a unique choice for $\xi_{i+1}^{\prime}$ with the desired properties. After transferring all triangles $\Delta\left(\zeta, \xi_{i}, \xi_{i+1}\right)$, the concatenation of the segments $\overline{\xi_{i}^{\prime} \xi_{i+1}^{\prime}}$ forms a (locally) geodesic path $\gamma$ of the metric length equal to that of $\overline{\xi \eta}$, i.e. $\leq \pi$. Since $B^{\prime}$ is a CAT(1) space, $\gamma$ is the geodesic segment $\overline{\xi^{\prime} \eta^{\prime}}$. By the construction, $\gamma$ has the same refined length as $\overline{\xi \eta}$. This concludes the proof in the spherical case.

(i) The same argument works in the Euclidean case, applying the result for spherical buildings of one dimension less.

As a consequence, we can define refined side length spaces $\mathcal{P}_{n}^{\text {ref }}\left(\left(E, W_{\text {aff }}\right)\right)$ respectively $\mathcal{P}_{n}^{r e f}((S, W))$ associated to Euclidean and spherical Coxeter complexes. They describe the possible refined side lengths of polygons in thick buildings modelled on these Coxeter complexes. To be consistent with earlier notation we may also write $\mathcal{P}_{n}^{r e f}\left(\Delta_{\text {sph }}\right)$ instead of $\mathcal{P}_{n}^{r e f}((S, W))$.

Our proof of the Transfer Theorem 3.2 allows us more generally to transfer polygons from buildings with smaller Weyl groups to buildings with larger Weyl 
groups. Suppose that

$$
\left(E, W_{a f f}\right) \hookrightarrow\left(E^{\prime}, W_{a f f}^{\prime}\right)
$$

is an embedding of Euclidean Coxeter complexes, i.e. a homothety $E \rightarrow E^{\prime}$ equivariant with respect to a monomorphism $W_{\text {aff }} \hookrightarrow W_{\text {aff }}^{\prime}$, cf. section 2.3. Our above argument yields

Addendum 3.3 (to 3.2). (i) The map

$$
\left((E \times E) / W_{a f f}\right)^{n} \rightarrow\left(\left(E^{\prime} \times E^{\prime}\right) / W_{a f f}^{\prime}\right)^{n}
$$

induced by the embedding of Euclidean Coxeter complexes (4) maps $\mathcal{P}_{n}^{\text {ref }}\left(\left(E, W_{\text {aff }}\right)\right)$ into $\mathcal{P}_{n}^{\text {ref }}\left(\left(E^{\prime}, W_{\text {aff }}^{\prime}\right)\right)$.

(ii) The analogous assertion for embeddings of spherical Coxeter complexes.

A variation of the transfer construction, namely the folding of polygons into apartments, is discussed in [KLM2].

\section{Polygons and Weighted Configurations at Infinity}

The Transfer Theorem 3.2 says that the possible refined side lengths for polygons in a thick Euclidean building depend only on the associated affine Coxeter complex. We now address our Main Theorem 1.1 and show that the unrefined $\Delta$-side lengths depend only on the spherical Coxeter complex. That is, we relate the $\Delta$-side lengths of polygons in Euclidean buildings with the same spherical Weyl group but whose affine Weyl groups may have different translation subgroups. Addendum 3.3 allows us to transfer polygons from buildings with smaller affine Weyl groups to buildings with larger ones. But to go in the other direction we have to pass through configurations at infinity.

We first introduce in section 4.1 a notion of stability for weighted configurations on spherical buildings which is motivated by (and consistent with, cf. [KLM1, Ch. 4]) Mumford stability in geometric invariant theory. In section 4.2 we explain how an oriented polygon in a Euclidean building $X$ gives rise to a collection of Gauss maps which can be regarded as weighted configurations on the spherical Tits building $\partial_{\text {Tits }} X$ at infinity, and prove the basic Lemma 4.3 that the arising configurations are semistable. The converse question when a semistable configuration $\psi$ on the Tits boundary $\partial_{\text {Tits }} X$ is the Gauss map of a polygon in $X$ amounts to a fixed-point problem for a certain weak contraction $\Phi_{\psi}: X \rightarrow X$. In section 4.3 we prove the existence of a fixed point in the special case when $X$ is a Euclidean building with one vertex, i.e. when it is isometric to the complete Euclidean cone over its Tits boundary. In section 4.4 we combine our results and prove the main theorems stated in the introduction.

4.1 Weighted configurations on spherical buildings and stability. Let $B$ be a spherical building. We denote the metric on $B$ by $\angle_{\text {Tits }}$ because spherical buildings appear in this paper usually as Tits boundaries. 
A collection of points $\xi_{1}, \ldots, \xi_{n} \in B$ and of weights $m_{1}, \ldots, m_{n} \geq 0$ determines a weighted configuration

$$
\psi:(\mathbb{Z} / n \mathbb{Z}, \nu) \rightarrow B
$$

on $B$. Here $\nu$ is the measure on $\mathbb{Z} / n \mathbb{Z}$ defined by $\nu(i)=m_{i}$, and the map $\psi$ sends $i$ to $\xi_{i}$. By composing $\psi$ with the natural accordion projection acc : $B \rightarrow \Delta_{\text {sph }}$ onto the associated spherical Weyl chamber $\Delta_{s p h}$, compare section 2.4, one obtains a map $(\mathbb{Z} / n \mathbb{Z}, \nu) \rightarrow \Delta_{\text {sph }}$. We call the corresponding point $h(\psi)=\left(h_{1}, \ldots, h_{n}\right)$ in $\Delta_{\text {euc }}^{n}$ the $\Delta$-weights of the configuration $\psi$, i.e. $h_{i}=m_{i} \cdot a c c\left(\xi_{i}\right)$. Recall that $\Delta_{\text {euc }}$ is defined as the complete Euclidean cone over $\Delta_{s p h}$.

The configuration $\psi$ yields, by pushing forward $\nu$, the measure $\mu=\sum m_{i} \delta_{\xi_{i}}$ on $B$. We defined its slope function on $B$ by

$$
\text { slope }_{\mu}=-\sum_{i \in \mathbb{Z} / n \mathbb{Z}} m_{i} \cos \angle_{T i t s}\left(\xi_{i}, \cdot\right) .
$$

Definition 4.1 (Stability). The measure $\mu$ on $B$ is called semistable if slope $_{\mu} \geq 0$ and stable if slope $e_{\mu}>0$ everywhere on $B$. The weighted configuration $\psi$ is called (semi)stable if the associated measure has this property.

In [KLM1] we also define nice semistable measures, see [KLM1, Def. 3.12]. This refinement of the notion of semistability amounts to saying that the associated measures $\mu$ are semistable and $\left\{\right.$ slope $\left._{\mu}=0\right\}$, if non-empty, is a sub-building. In the case when $B$ is the Tits boundary of a symmetric space $X$, it turns out that $\left\{\right.$ slope $\left._{\mu}=0\right\}$ is the Tits boundary of a totally-geodesic subspace of $X$. See [KLM1] for the detailed discussion.

EXAMPle 4.2. Let $B$ be a spherical building of dimension 0 . Then a measure $\mu$ on $B$ is stable iff all atoms have mass $<\frac{1}{2}|\mu|$, is semistable iff all atoms have mass $\leq \frac{1}{2}|\mu|$, and is nice semistable iff it is either stable or consists of two atoms of the same mass.

The terminology slope becomes clear when one considers spherical buildings as Tits boundaries. If $X$ is a Euclidean building (or a symmetric space of noncompact type) then we can associate with a measure $\mu=\sum m_{i} \delta_{\xi_{i}}$ on $\partial_{\text {Tits }} X$ its weighted Busemann function

$$
b_{\mu}:=\sum_{i \in \mathbb{Z} / n \mathbb{Z}} m_{i} b_{\xi_{i}},
$$

on $X$, cf. the definition of Busemann functions in section 2.2 and the discussion of their asymptotics. The function $b_{\mu}$ is well defined up to an additive constant and convex. For any ideal point $\eta \in \partial_{\text {Tits }} X$ and any unit speed geodesic ray $\rho:[0, \infty) \rightarrow X$ asymptotic to $\eta$, the formula (3) implies that

$$
\operatorname{slope}_{\mu}(\eta)=\lim _{t \rightarrow \infty} \frac{b_{\mu}(\rho(t))}{t},
$$

i.e. slope $_{\mu}(\eta)$ computes the asymptotic slope of $b_{\mu}$ in the direction $\eta$. 
4.2 From polygons to configurations: Gauss maps. Let $X$ be a Euclidean building or a symmetric space of noncompact type. We now relate polygons in $X$ and weighted configurations on the spherical Tits building $\partial_{\text {Tits }} X$ at infinity.

Consider a polygon $P=x_{1} x_{2} \ldots x_{n}$ in $X$, i.e. a map $\mathbb{Z} / n \mathbb{Z} \rightarrow X$. The distances $m_{i}=d\left(x_{i-1}, x_{i}\right)$ determine a measure $\nu$ on $\mathbb{Z} / n \mathbb{Z}$ by putting $\nu(i)=m_{i}$. The polygon $P$ gives rise to a collection Gauss $(P)$ of Gauss maps

$$
\psi: \mathbb{Z} / n \mathbb{Z} \longrightarrow \partial_{\text {Tits }} X
$$

by assigning to $i$ an ideal point $\xi_{i} \in \partial_{\text {Tits }} X$ so that the ray $\overline{x_{i-1} \xi_{i}}$ passes through $x_{i}$. This construction, in the case of hyperbolic plane, already appears in the letter of Gauss to W. Bolyai $[\mathrm{G}]$. Taking into account the measure $\nu$, we view the maps $\psi:(\mathbb{Z} / n \mathbb{Z}, \nu) \rightarrow \partial_{\text {Tits }} X$ as weighted configurations on $\partial_{\text {Tits }} X$. Their $\Delta$-weights equal the $\Delta$-side lengths of the polygon $P$.

Note that if $X$ is a Riemannian symmetric space and the $m_{i}$ are non-zero, there is a unique Gauss map for $P$ because geodesic segments are uniquely extendible to complete geodesics. On the other hand, if $X$ is a Euclidean building then, due to the branching of geodesics, there are in general several Gauss maps. However, the corresponding weighted configurations have the same $\Delta$-weights.

The following observation is basic for us and explains why the notion of semistability is useful in studying polygons.

Lemma 4.3 (Semistability of Gauss maps). The pushed forward measures $\mu=\psi_{*} \nu$ are semistable.

Proof. Let $\eta \in \partial_{\text {Tits }} X$ and let $\gamma_{i}:\left[0, m_{i}\right] \rightarrow X$ be a unit speed parametrization of the geodesic segment $\overline{x_{i-1} x_{i}}$. Then the Busemann function $b_{\eta}$ is one-sided differentiable along $\gamma_{i}$ with derivative

$$
\frac{d}{d t^{+}}\left(b_{\eta} \circ \gamma_{i}\right)(t)=-\cos \angle_{\gamma_{i}(t)}\left(\xi_{i}, \eta\right) \leq-\cos \angle_{\text {Tits }}\left(\xi_{i}, \eta\right),
$$

cf. the formula (2) in section 2.2 for the directional derivatives of Busemann functions. Integrating along $\gamma_{i}$ we obtain

$$
b_{\eta}\left(x_{i}\right)-b_{\eta}\left(x_{i-1}\right) \leq-m_{i} \cdot \cos \angle_{T i t s}\left(\xi_{i}, \eta\right)
$$

and summation over all sides yields

$$
0 \leq-\sum_{i \in \mathbb{Z} / n \mathbb{Z}} m_{i} \cdot \cos \angle_{\text {Tits }}\left(\xi_{i}, \eta\right)=\operatorname{slope~}_{\mu}(\eta)
$$

confirming the semistability.

REMARK 4.4. If $X$ is a Riemannian symmetric space one can prove the sharper result that the weighted configurations on $\partial_{\text {Tits }} X$ arising as Gauss maps of closed polygons in $X$ are nice semistable, see [KLM1, Lem. 5.5]. 


\subsection{From configurations to polygons: fixed points for weak contractions.}

Let $X$ be a Euclidean building or a symmetric space of noncompact type. We are now interested in finding polygons with prescribed Gauss map. Such polygons will correspond to the fixed points of a certain weakly contracting self map of $X$.

For $\xi \in \partial_{\text {Tits }} X$ and $t \geq 0$, we define the map $\phi_{\xi, t}: X \rightarrow X$ by sending $x$ to the point at distance $t$ from $x$ on the geodesic ray $\overline{x \xi}$. Since $X$ is nonpositively curved, the function $\delta: t \mapsto d\left(\phi_{\xi, t}(x), \phi_{\xi, t}(y)\right)$ is convex. It is also bounded because the rays $\overline{x \xi}$ and $\overline{y \xi}$ are asymptotic, and hence it is monotonically non-increasing in $t$. This means that the maps $\phi_{\xi, t}$ are weakly contracting, i.e. they have Lipschitz constant 1. For a weighted configuration $\psi:(\mathbb{Z} / n \mathbb{Z}, \nu) \rightarrow \partial_{\text {Tits }} X$ we define the weak contraction

$$
\Phi=\Phi_{\psi}: X \longrightarrow X
$$

as the composition $\phi_{\xi_{n}, m_{n}} \circ \cdots \circ \phi_{\xi_{1}, m_{1}}$. The fixed points of $\Phi$ are the $n$-th vertices of closed polygons $P=x_{1} \ldots x_{n}$ with $\psi \in \operatorname{Gauss}(P)$.

Regarding the existence of fixed points for $\Phi$, we will only need the special case of buildings with one vertex, that is, of complete Euclidean cones over spherical buildings.

Proposition 4.5. Suppose that $X$ is a Euclidean building with one vertex and that $\psi$ is a semistable weighted configuration on $\partial_{\text {Tits }} X$. Then the weak contraction $\Phi_{\psi}: X \rightarrow X$ has a fixed point.

The following auxiliary result may be of independent interest. It extends Cartan's fixed-point theorem for isometric actions on nonpositively curved spaces with bounded orbits. Note that we do not need to assume local compactness.

Lemma 4.6. Let $Y$ be a Hadamard space and $\Phi: Y \rightarrow Y$ a 1-Lipschitz self map. If the forward orbits $\left(\Phi^{n} y\right)_{n \geq 0}$ are bounded then $\Phi$ has a fixed point in $Y$.

Proof. Consider an orbit $y_{n}=\Phi^{n} y_{0}$ of a point $y_{0} \in Y$ and define the distance from its "tail" by

$$
r(y):=\limsup _{n \rightarrow \infty} d\left(y_{n}, y\right) .
$$

Note that $r$ inherits from the distance function the convexity and the 1-Lipschitz continuity. The assumption that $\Phi$ is 1-Lipschitz implies

$$
r(\Phi y)=\limsup _{n \rightarrow \infty} d\left(y_{n}, \Phi y\right)=\limsup _{n \rightarrow \infty} d\left(\Phi y_{n-1}, \Phi y\right) \leq \limsup _{n \rightarrow \infty} d\left(y_{n-1}, y\right)=r(y)
$$

that is,

$$
r \circ \Phi \leq r
$$

It suffices to show that $r$ has a unique minimum since this would then be a fixed point of $\Phi$. We denote

$$
\rho:=\inf _{Y} r
$$

For $\epsilon>0$, let $y, y^{\prime}$ be points with $r(y)=r\left(y^{\prime}\right)<\rho+\epsilon$. Then there exists $n_{0}$ such that for $n \geq n_{0}$ we have

$$
d\left(y_{n}, y\right), d\left(y_{n}, y^{\prime}\right)<\rho+\epsilon \text {. }
$$


On the other hand, let $m$ be the midpoint of $\overline{y y^{\prime}}$. Since $r(m) \geq \rho$ we have

$$
d\left(y_{n}, m\right)>\rho-\epsilon
$$

for infinitely many $n$. For these $n$ we apply triangle comparison to the triangle $\Delta\left(y, y^{\prime}, y_{n}\right)$ with Euclidean plane as model space, cf. section 2.1. For the comparison triangle $\Delta\left(\tilde{y}, \tilde{y}^{\prime}, \tilde{y}_{n}\right)$ in Euclidean plane we have the parallelogram identity,

$$
d\left(\tilde{y}, \tilde{y}^{\prime}\right)^{2}+4 d\left(\tilde{y}_{n}, \tilde{m}\right)^{2}=2\left(d\left(\tilde{y}_{n}, \tilde{y}\right)^{2}+d\left(\tilde{y}_{n}, \tilde{y}^{\prime}\right)^{2}\right) .
$$

By the chord comparison we have $d\left(y_{n}, m\right) \leq d\left(\tilde{y}_{n}, \tilde{m}\right)$ and obtain the inequality

$$
d\left(y, y^{\prime}\right)^{2}+4 \underbrace{d\left(y_{n}, m\right)^{2}}_{>\rho-\epsilon} \leq 2(\underbrace{d\left(y_{n}, y\right)^{2}}_{<\rho+\epsilon}+\underbrace{d\left(y_{n}, y^{\prime}\right)}_{<\rho+\epsilon})^{2}
$$

and

$$
d\left(y, y^{\prime}\right)^{2}<16 \rho \epsilon+8 \epsilon^{2}
$$

It follows that any sequence $\left(z_{k}\right)$ in $Y$ with $r\left(z_{k}\right) \searrow \rho$ must be a Cauchy sequence. Then completeness of $Y$ implies that $r$ has a minimum, and the minimum must be unique.

Proof of Proposition 4.5. The building $X$ is isometric to the complete Euclidean cone Cone $\left(\partial_{\text {Tits }} X\right)$ over its Tits boundary. We denote the unique vertex of $X$ by $o$.

Due to the conicality of $X$ the contraction $\Phi$ has a fairly simple geometry. Let $\sigma$ be a simplex in $\partial_{\text {Tits }} X$ and let $V$ be the corresponding face of $X$, i.e. the Euclidean sector with tip $o$ and ideal boundary $\sigma$. Let $\xi \in \partial_{\text {Tits }} X$ and $t \geq 0$. By the building axioms, for every face $W \supseteq V$ of $X$ there exists a (maximal) flat $F \subset X$ with $W \subset F$ and $\xi \in \partial_{\text {Tits }} F$. The map $\phi_{\xi, t}$ restricts on $F$ to a translation and we have

$$
b_{\eta}\left(\phi_{\xi, t} x\right)-b_{\eta}(x)=-t \cdot \cos \angle_{T i t s}(\xi, \eta)
$$

for $\eta \in \partial_{\text {Tits }} F$ and $x \in F$. Since we may vary $W$, the equation (12) holds for all $\eta \in \sigma$ and $x \in \operatorname{star}(V)$. We define

$$
\check{s} \operatorname{tar}(V):=\left\{x \in \operatorname{star}(V): B_{|\mu|}(x) \subset \operatorname{star}(V)\right\}
$$

where $|\mu|$ denotes the total mass of $\mu$ and $B_{|\mu|}(x)$ denotes the metric ball of radius $|\mu|$ centered at $x$, i.e. štar $(V)$ consists of those points in $\operatorname{star}(V)$ which have at least distance $|\mu|$ from its boundary. Since $\Phi$ has displacement $\leq|\mu|$, we have $\Phi(\check{s} \operatorname{tar}(V)) \subset \operatorname{star}(V)$ and

$$
b_{\eta}(\Phi x)-b_{\eta}(x)=\operatorname{slope}_{\mu}(\eta)
$$

for $\eta \in \sigma$ and $x \in \check{s} \operatorname{tar}(V)$.

We may use the Busemann functions to measure the distance from the vertex $o$. With the normalization $b_{\eta}(o)=0$ we have $d(o, \cdot)=\max _{\eta \in \partial_{\text {Tits }} X}\left(-b_{\eta}\right)$. For technical reasons we discretize as follows. We fix a finite subset $F \subset \Delta_{s p h}$ in the spherical 
model Weyl chamber which has the property: If $\eta \in \Delta_{\text {sph }}$ and $\zeta \in F$ such that $\angle_{\text {Tits }}(\eta, \zeta) \leq 2 \angle_{\text {Tits }}\left(\eta, \zeta^{\prime}\right)$ for all $\zeta^{\prime} \in F$ then $\eta$ has distance $>\epsilon$ from all faces of $\Delta_{s p h}$ which do not contain $\zeta$ in their closure. Let $A \subset \partial_{\text {Tits }} X$ be the discrete subset consisting of all points with types in $F$, that is the inverse image of $F$ under the canonical projection acc $: \partial_{\text {Tits }} X \rightarrow \Delta_{s p h}$. By the construction $A$ satisfies the following: If $\eta \in \partial_{\text {Tits }} X$ and $\zeta \in A$ such that $\angle_{\text {Tits }}(\eta, \zeta) \leq 2 \angle_{\text {Tits }}\left(\eta, \zeta^{\prime}\right)$ for all $\zeta^{\prime} \in A$ then $\eta$ has distance $>\epsilon$ from all faces of $\partial_{\text {Tits }} X$ which do not contain $\zeta$ in their closure, i.e. $B_{\epsilon}(\eta) \subset \operatorname{star}\left(\sigma_{\zeta}\right)$ where $\sigma_{\zeta}$ denotes the simplex of $\partial_{\text {Tits }} X$ containing $\zeta$ as an interior point.

As an approximation to $d(o, \cdot)$ we use the function

$$
f:=\max _{\zeta \in A}\left(-b_{\zeta}\right)
$$

This function has bounded sublevel sets, and according to Lemma 4.6 we are done once we can show that $\Phi$ preserves some non-empty sublevel set.

Let $r>0$ and $x \in X$ with $d(o, x)>r$. For any $\zeta \in A$, we wish to show that $-b_{\zeta}(\Phi x) \leq f(x)$. Suppose first that $\angle_{o}(x, \zeta) \leq 2 \angle_{o}\left(x, \zeta^{\prime}\right)$ for all $\zeta^{\prime} \in A$. Then, if $r$ has been chosen sufficiently large, we have $x \in \check{s} \operatorname{tar}\left(V_{\zeta}\right)$ where $V_{\zeta}$ denotes the face of $X$ with ideal boundary $\sigma_{\zeta}$. Applying (13) and using that $\mu$ is semistable we obtain

$$
-b_{\zeta}(\Phi x) \leq-b_{\zeta}(x) \leq f(x)
$$

in this case. On the other hand, if $\angle_{o}(x, \zeta)>2 \angle_{o}\left(x, \zeta^{\prime}\right)$ with $\zeta^{\prime} \in A$ and

$$
\angle_{o}\left(x, \zeta^{\prime}\right)=\min _{\zeta^{\prime \prime} \in A} \angle_{o}\left(x, \zeta^{\prime \prime}\right),
$$

then

$$
f(x)=-b_{\zeta^{\prime}}(x)>-b_{\zeta}(x)+|\mu|,
$$

again if $r$ has been chosen large enough. So

$$
-b_{\zeta}(\Phi x) \leq-b_{\zeta}(x)+|\mu| \leq f(x)
$$

in this case as well. We conclude that $f(\Phi x) \leq f(x)$ if $d(o, x)$ is sufficiently large. Since $\Phi$ is 1-Lipschitz it follows that it preserves $\{f \leq R\}$ for large enough $R>0$.

REMARK 4.7. One can show that, if $X$ is a Euclidean building and $\psi$ semistable or if $X$ is a symmetric space of noncompact type and $\psi$ nice semistable, then $\Phi_{\psi}$ has a fixed point. The case of Euclidean buildings which are not necessarily locally compact is due to Andreas Balser [Ba]. The case of symmetric spaces is proven in [KLM1].

\subsection{Proofs of the main results.}

Proof of Theorem 1.2. That the existence of polygons implies the existence of configurations follows from the semistability of Gauss maps, cf. Lemma 4.3.

The converse has been proven in Proposition 4.5 for Euclidean buildings with one vertex. We deduce it for an arbitrary Euclidean building $X$ by using the 
Addendum 3.3 to the Transfer Theorem 3.2. Namely, suppose that $h$ is the $\Delta$ weight of a semistable configuration on $\partial_{\text {Tits }} X$. Then by Proposition 4.5 there exist closed polygons with $\Delta$-side lengths $h$ in the Euclidean building $C o n e\left(\partial_{\text {Tits }} X\right)$. Now the Euclidean Coxeter complex of $C$ one $\left(\partial_{\text {Tits }} X\right)$ embeds into the Euclidean Coxeter complex of $X$; namely if $\left(E, W_{a f f}\right)$ denotes the Euclidean Coxeter complex attached to $X$ then the Euclidean Coxeter complex attached to Cone $\left(\partial_{\text {Tits }} X\right)$ is isomorphic to $\left(E, W_{s p h}\right)$. Addendum 3.3 therefore implies the existence of polygons with $\Delta$-side lengths $h$ in $X$.

Notice that our argument does not produce a polygon having the given configuration as a Gauss map, compare remark 4.7.

Proof of Theorem 1.4. Consider two thick spherical buildings $B$ and $B^{\prime}$ modelled on the same spherical Coxeter complex. The thick Euclidean buildings $C o n e(B)$ and Cone $\left(B^{\prime}\right)$ are then modelled on the same Euclidean Coxeter complex. The Transfer Theorem 3.2 implies that in both spaces the same refined side lengths occur for closed polygons; this immediately implies the same assertion for the $\Delta$-side lengths. It follows from Theorem 1.2 that the same $\Delta$-weights occur for semistable weighted configurations on $B$ and $B^{\prime}$.

Proof of Main Theorem 1.1. Consider two thick Euclidean buildings $X$ and $X^{\prime}$, and suppose that their spherical Tits buildings $\partial_{\text {Tits }} X$ and $\partial_{\text {Tits }} X^{\prime}$ at infinity are modelled on the same spherical Coxeter complex. The spherical buildings are thick as well, and the claim therefore follows from Theorems 1.2 and 1.4.

\section{References}

[B] W. Ballmann, Lectures on Spaces of Nonpositive Curvature (with an appendix by Misha Brin, DMV Seminar 25, Birkhäuser Verlag, Basel, 1995.

[Ba] A. BALSER, Polygons with prescribed Gauss map in Hadamard spaces and Euclidean buildings, Canad. Math. Bull. 49:3 (2006), 321-336.

[BrH] M. Bridson, A. Haefliger, Metric Spaces of Non-positive Curvature, Grundlehren 319, Springer, 1999.

[Bro] K. Brown, Buildings, Springer, 1989.

[BuBI] D. Burago, Y. Burago, S. Ivanov, A Course in Metric Geometry, Graduate Studies in Mathematics 33, AMS, 2001.

[D] M. DAvis, Buildings are CAT(0), in "Geometry and Cohomology in Group Theory (Durham, 1994)", London Math. Soc. Lecture Note Ser. 252, Cambridge Univ. Press, Cambridge (1998), 108-123.

[E] P. Eberlein, Geometry of Nonpositively Curved Manifolds, Chicago Lectures in Mathematics, University of Chicago Press, Chicago, IL, 1996.

[G] C.F. Gauss, Letter to W. Bolyai, Collected Works 8, 222-223.

[KLM1] M. Kapovich, B. Leeb, J. Millson, Convex functions on symmetric spaces, side lengths of polygons and the stability inequalities for weighted configurations at infinity, Journal of Differential Geometry 81 (2009), 297-354.

[KLM2] M. Kapovich, B. Leeb, J. Millson, The Generalized Triangle Inequalities in Symmetric Spaces and Buildings with Applications to Algebra, Memoirs of AMS 192 (2008). 
[KlL] B. Kleiner, B. LeEB, Rigidity of quasi-isometries for symmetric spaces and Euclidean buildings, Publ. Math. Inst. Hautes Études Sci. 86 (1997), 115-197.

[L] B. LeEB, A Characterization of Irreducible Symmetric Spaces and Euclidean Buildings of Higher Rank by Their Asymptotic Geometry, Bonner Mathematische Schriften 326, Bonn, 2000.

[OS] L. O'Shea, R. SjamaAr, Moment maps and Riemannian symmetric pairs, Math. Ann. 317 (2000), 415-457.

[R] M. Ronan, Lectures on Buildings, Perspectives in Mathematics 7, Academic Press, 1989.

[S] R. Scharlau, A structure theorem for weak buildings of spherical type, Geom. Dedicata 24 (1987), 77-84.

Michael Kapovich, Department of Mathematics, University of California, Davis, CA 95616, USA kapovich@math.ucdavis.edu

Bernhard Leeb, Mathematisches Institut, Universität München, Theresienstrasse 39, D80333 München, Germany

b. I@lmu.de

John Millson, Department of Mathematics, University of Maryland, College Park, MD 20742, USA

jjm@math.umd.edu

Received: June 10, 2008

Accepted: August 14, 2008

Open Access This article is distributed under the terms of the Creative Commons Attribution Noncommercial License which permits any noncommercial use, distribution, and reproduction in any medium, provided the original author(s) and source are credited. 